\title{
Brittle-tough transition in nylon-rubber blends: effect of rubber concentration and particle size
}

\author{
R. J. M. Borggreve, R. J. Gaymans, J. Schuijer and J. F. Ingen Housz \\ University of Twente, PO Box 2177500 AE Enschede, The Netherlands \\ (Received 16 January 1987; accepted 18 March 1987)
}

\begin{abstract}
Blends of nylon- 6 and EPDM-rubber were prepared with various rubber contents $(0-20 \mathrm{wt} \%)$ and particle sizes $(0.3-1.6 \mu \mathrm{m})$. The effects of rubber concentration and particle size on the tensile modulus, torsion modulus, yield stress and notched impact strength of the blends were studied. Blend structures and fracture surfaces were investigated by scanning electron microscopy. Rubber particles induce a sharp brittle-tough transition which is independent of the glass transition temperature of the nylon matrix. The brittle-tough transition temperature for notched Izod impact tests shifts to lower values when the rubber content is increased or the particle size is decreased. A correlation was found between the brittle-tough temperature and the interparticle distance. Two deformation modes were observed: voiding and shear yielding. Particle size and interfacial adhesion affect neither the yield stress nor the modulus of the blends.
\end{abstract}

(Keywords: nylon-rubber blend; impact toughness; rubber concentration; particle size; interparticle distance; interfacial adhesion; deformation mode)

\section{INTRODUCTION}

In rubber modified ductile polymers such as polyamides and polypropylene a sharp brittle-tough transition is observed when experimental conditions (temperature, strain rate, specimen geometry) or material parameters (rubber concentration, particle size, interparticle distance) are varied ${ }^{1-7}$.

$\mathrm{Jang}^{4}$ attributes the transition from brittle to tough fracture behaviour in polypropylene-rubber blends to a competition between two deformation mechanisms: crazing and shear yielding. When the craze initiation stress $\sigma_{\mathrm{cr}}$ is lower than the shear initiation stress $\sigma_{\mathrm{sh}}$ the failure mode is crazing and brittle fracture occurs. Otherwise, when $\sigma_{\mathrm{sh}}<\sigma_{\mathrm{cr}}$ shear yielding will be the main deformation mechanism and large energy absorption takes place, resulting in a tough fracture.

According to this theory each test and material parameter may affect the crazing and shear banding process to different degrees. The test conditions where $\sigma_{\mathrm{cr}}=\sigma_{\mathrm{sh}}$ define the brittle-tough (BT) transition of the material. On the other hand, $\mathrm{Wu}^{5}$ found that in both brittle and tough fractures of nylon-rubber blends about $75 \%$ of the energy dissipation is due to matrix yielding and $25 \%$ is due to matrix crazing. The difference in impact energy arises from the different size of the energy dissipation zone.

According to $\mathrm{Wu}$, tough fracture is running entirely within the nylon matrix since rubber particles were not observed on a tough fracture surface. In the case of brittle fracture, on the other hand, rubber particles were observed on the fracture surface, suggesting that the crack path goes through the particle or the particle-matrix interface.

Hobbs $^{6}$ developed a theory that the BT transition depends on the number of defined interactions of stress concentration fields around rubber particles. In nylon- rubber blends, as well as in polypropylene-rubber blends, the shift in BT transition could be predicted by counting the number of interactions.

The BT transition would only depend on rubber concentration and particle size since these parameters determine the number of interactions.

$\mathrm{Wu}^{7}$ stated recently that the material parameter which controls the BT transition of nylon-rubber blends is the interparticle distance $(I D)$. If $I D$ is greater than the critical value $\left(I D_{\mathrm{c}}\right)$ the fracture mode is brittle. If $I D<I D_{\mathrm{c}}$ the fracture behaviour will be tough. $I D_{\mathrm{c}}$ is independent of particle size and rubber volume fraction and is characteristic of a given matrix. Interfacial chemical bonding is not necessary and van der Waals attraction gives sufficient adhesion for toughening.

Merle $^{8}$ and Adams ${ }^{9}$ studied nylon-rubber blends with instrumented impact testing. From load-time curves they deduced that energy absorption in brittle fracture only takes place at crack initiation whereas in tough fractures large energy absorption also occurs during crack propagation.

An interesting 'study on Charpy impact fractures of (toughened) poly(butylene terephthalate) using high speed photography was performed by Polato ${ }^{10}$. During tough fracture, the fracture tip radius grows due to extensive crack blunting. In brittle fracture the crack tip remains very sharp during crack propagation.

Because pure, dry nylon behaves in a brittle fasion only at high strain rates we studied the BT transition of nylonrubber blends during notched impact testing with varying temperature. The impact behaviour strongly depends on the specimen geometry and it is emphasized that the reported impact energies and BT temperatures are closely bound up with the chosen impact test.

It is known that the orientation of macromolecules due to the injection moulding process influences the fracture behaviour of polymers. In a direction normal to injection, 
anisotropic materials are less tough than in a direction parallel to injection ${ }^{11}$. All samples used in our study are injection moulded and tested in the same way, so ruling out anisotropy and specimen geometry as variables.

Material parameters which may affect the notched impact strength and the BT transition of nylon rubber blends are: (a) rubber concentration; (b) rubber particle size; (c) shear modulus of the rubber; (d) interfacial adhesion; and (e) nylon matrix structure. In this work we consider the influence of the first two factors.

The structure of the matrix does not change significantly with type, size and amount of rubber ${ }^{5,7}$ and is regarded as constant when varying the rubber concentration and particle size.

A suitable blend for studying the impact behaviour is nylon-6/EPDM. Nylon and rubber are chemically coupled with maleic anhydride. The anhydride is first grafted onto the rubber, then during blending a reaction between anhydride and amine may occur, as described by Cimmino ${ }^{12}$.

\section{EXPERIMENTAL}

\section{Materials}

The matrix used was nylon-6 (Akulon M258) obtained from Akzo, with $\eta_{\text {rel }}=5.8$ in $96 \% \mathrm{H}_{2} \mathrm{SO}_{4}$. The rubber was EPDM (ethylene-propylene-dicyclopentadiene, 65:34:1, wt $\%$; Keltan 740, supplied by DSM) grafted with maleic anhydride. The grafting reaction was carried out in a Brabender $19 \mathrm{~mm}$ extruder in the presence of bis(tbutylperoxyisopropyl)benzene (Perkadox 14, supplied by Akzo Chemie). The extent of grafting was measured with both infra-red spectroscopy and potentiometric titration.

\section{Blend preparation}

The blends were prepared by compounding nylon- 6 with the modified EPDM on a Kautex $40 \mathrm{~mm}$ singlescrew extruder fitted with a $10 \mathrm{~cm}$ long cavity transfer mixing (CTM) head ${ }^{13}$. Two methods of blending were performed.

(i) When varying the rubber concentration, a two-step procedure was followed. (1) A master batch of $20 \mathrm{wt} \%$ EPDM was prepared in the Kautex $40 \mathrm{~mm}$ extruder with the CTM, at a screw speed of $50 \mathrm{rev} / \mathrm{min}$ and barrel temperatures $225-225-225-225^{\circ} \mathrm{C}$. (2) The master batch was diluted to lower concentrations of rubber in the Brabender extruder at elevated temperatures (low shear).

(ii) Rubber particle size was changed at constant rubber fraction by varying the barrel temperatures of the Kautex extruder.

The blends were injection moulded on an Arburg Allrounder 221-35-250 to samples for impact testing and tensile tests.

\section{Material testing}

The samples were dried for $24 \mathrm{~h}$ at $110^{\circ} \mathrm{C}$ in a vacuum oven before testing.

Notched Izod impact strength was measured (ISO 180/1A) using a Zwick impact testing machine in a temperature range from $-40^{\circ} \mathrm{C}$ up to $80^{\circ} \mathrm{C}$. The average values of at least five tests are reported and are typical for the specimen geometry used in the test (Figure 1).

Tensile tests were performed on an Instron machine with a drawing speed of $250 \% \mathrm{~min}^{-1}$ at room temperature. The samples were dumb-bell shaped (DIN 53455 , specimen type 4).

Shear modulus and glass transition temperatures of the blends were measured with a Myrenne torsion apparatus ATM 3 at a frequency of $1 \mathrm{~Hz}$ and a heating rate of $0.5^{\circ} \mathrm{C} \min ^{-1}$.

\section{Microscopy}

To determine the rubber particle size, smooth surfaces of the samples were prepared using a Jung microtome, equipped with a glass knife and a liquid nitrogen cooling unit. Rubber was extracted from the surface by etching with boiling xylene overnight.

Fracture surfaces were either etched overnight with boiling xylene or stained for one week in $1 \%$ aqueous $\mathrm{OsO}_{4}$ to make the rubber distinguishable. The samples were furnished with a gold layer and examined with a Jeol scanning electron microscope. Particle size distribution was obtained from the photomicrographs.

\section{RESULTS AND DISCUSSION}

Table 1 gives the type of rubber, rubber content and particle size of the blends used in this work. In this study, the so-called weight-average particle size is used $\left(d_{\mathrm{w}}=\Sigma n_{i} d_{i}^{2} / \Sigma n_{i} d_{i}\right)$, which was found to have a better correlation with impact properties than the number average particle size ${ }^{14}\left(d_{\mathrm{n}}=\Sigma n_{i} d_{i} / \Sigma n_{i}\right)$.

From the holes in the surface the real particle size distribution can be calculated ${ }^{15}$. However, the proposed procedures turned out to be inaccurate when the distribution is broad ${ }^{14}$. Omitting this correction would not affect the essential features of the results.

The rubber particle sizes of blends $A$ are nearly constant, which means that neither a further reduction nor coalescence of the particles has taken place during the dilution step and the injection moulding process. This is in agreement with Elmendorp's results showing that coalescence during blending of two polymer melts is prevented when there is sufficient interfacial adhesion between the dispersion and the matrix phase ${ }^{16}$.

Compared to blends A, the particle sizes of blends B are considerably larger. A larger interfacial tension between nylon and rubber due to the lower grafting degree of maleic anhydride on EPDM results in larger rubber domains ${ }^{17}$. The particle sizes of blends $B$ are not constant because coalescence due to low interfacial adhesion

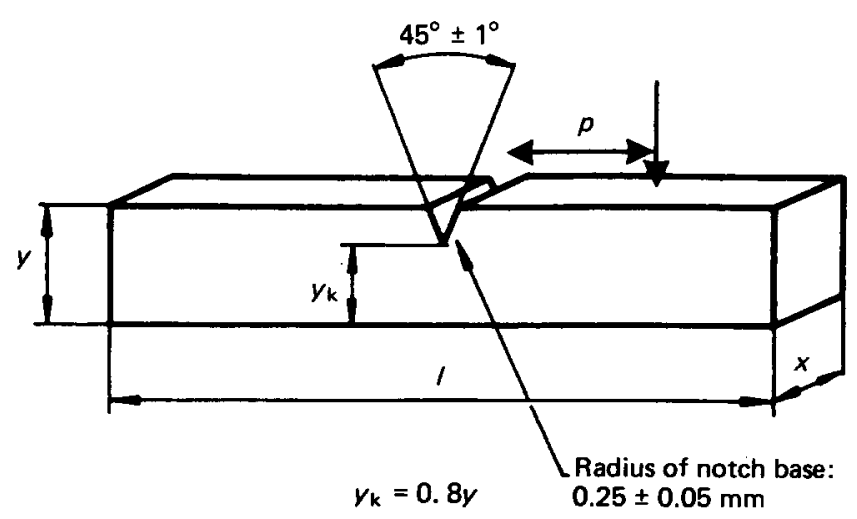

Figure 1 Geometry of the notched Izod specimen. $l=80 \mathrm{~mm}$; $y=10 \mathrm{~mm} ; x=4 \mathrm{~mm} . p=$ point of impact, $22 \mathrm{~mm}$ 
Brittle-tough transition in nylon-rubber blends: $R$. J. M. Borggreve et al.

Table 1 Blends of nylon-6 and EPDM rubber with varying maleic anhydride content, rubber content and weight-average particle size

\begin{tabular}{|c|c|c|c|c|}
\hline \multirow{2}{*}{$\begin{array}{l}\text { Blend } \\
\text { code }\end{array}$} & \multirow{2}{*}{$\begin{array}{l}\text { Maleic } \\
\text { anhydride } \\
\text { content } \\
(\mathrm{wt} \%)\end{array}$} & \multicolumn{2}{|c|}{ Rubber content } & \multirow{2}{*}{$\begin{array}{l}\text { Particle } \\
\text { size } \\
(\mu \mathrm{m})\end{array}$} \\
\hline & & $(w t \%)$ & $\left(\mathrm{vol}^{\%}\right)$ & \\
\hline 0 & - & 0 & 0 & - \\
\hline \multicolumn{5}{|c|}{ Blend method I } \\
\hline A.1 & 0.40 & 2 & 2.6 & 0.36 \\
\hline A. 2 & 0.40 & 5 & 6.4 & 0.38 \\
\hline A. 3 & 0.40 & 8 & 10.5 & 0.29 \\
\hline A.4 & 0.40 & 10 & 13.0 & 0.29 \\
\hline A. 5 & 0.40 & 15 & 19.6 & 0.33 \\
\hline A. 6 & 0.40 & 20 & 26.1 & 0.32 \\
\hline B. 1 & 0.10 & 2 & 2.6 & 0.52 \\
\hline B. 2 & 0.10 & 5 & 6.4 & 0.92 \\
\hline B. 3 & 0.10 & 8 & 10.5 & 0.78 \\
\hline B.4 & 0.10 & 10 & 13.0 & 0.87 \\
\hline B.5 & 0.10 & 15 & 19.6 & 1.05 \\
\hline B.6 & 0.10 & 20 & 26.1 & 1.94 \\
\hline \multicolumn{5}{|c|}{ Blend method II } \\
\hline C.1 & 0.25 & 20 & 26.1 & 0.48 \\
\hline C. 2 & 0.25 & 20 & 26.1 & 0.57 \\
\hline C. 3 & 0.25 & 20 & 26.1 & 0.94 \\
\hline C. 4 & 0.25 & 20 & 26.1 & 1.14 \\
\hline C.5 & 0.25 & 20 & 26.1 & 1.20 \\
\hline C. 6 & 0.25 & 20 & 26.1 & 1.59 \\
\hline
\end{tabular}

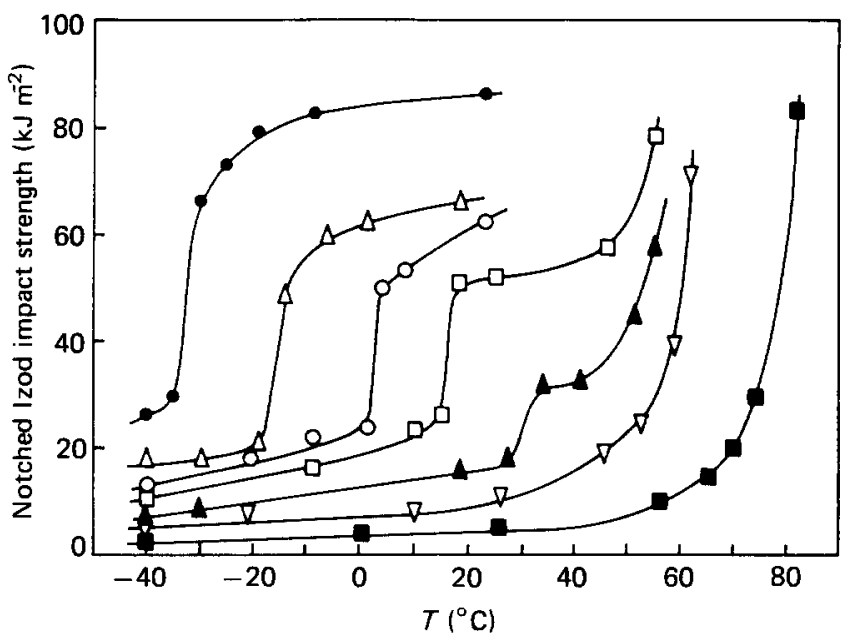

Figure 2 Notched Izod impact strength versus temperature of nylon-6 and blends $\mathrm{A}$. Particle size and interfacial adhesion are constant. Rubber concentration $(\mathrm{vol} \%): \square, 0 ; \nabla, 2.6 ; \mathbf{\Lambda}, 6.4 ; \square, 10.5 ; \bigcirc, 13.0 ; \triangle, 19.6$; , 26.1

becomes more intense with increasing rubber concentration.

The particle sizes of blends $\mathrm{C}$ increase when higher extrusion temperatures are applied.

\section{Effect of rubber concentration}

Rubber properties, interfacial adhesion and particle size of series A blends are constant. Figure 2 illustrates the influence of rubber concentration on the notched impact strength of nylon-rubber blends over a wide range of temperatures.

The notched impact strength of nylon-6 increases dramatically when the temperature exceeds the $T_{\mathrm{g}}$ of nylon-6. When the amorphous phase of the nylon changes from a glassy state into a rubbery state, the yield strength of the nylon is lowered by a factor 4 while the tensile strength changes by some $30 \%$ and thus excessive yielding can take place, resulting in a rapid increase in toughness ${ }^{18,19}$. Introducing rubber particles into the matrix creates a second brittle-tough transition. This transition is dispersion induced and discontinuous, i.e. a sudden increase in impact strength over a small temperature range.

The difference between a tough and a brittle fracture is also clear when studying the fracture surface: when the sample breaks in the brittle way, stress whitening can only be observed at the notch tip; in tough fracture, stress whitening takes place over the whole surface.

Figure 3 presents storage and loss moduli of the A blends. The maxima in the $G^{\prime \prime}$-temperature curve do not

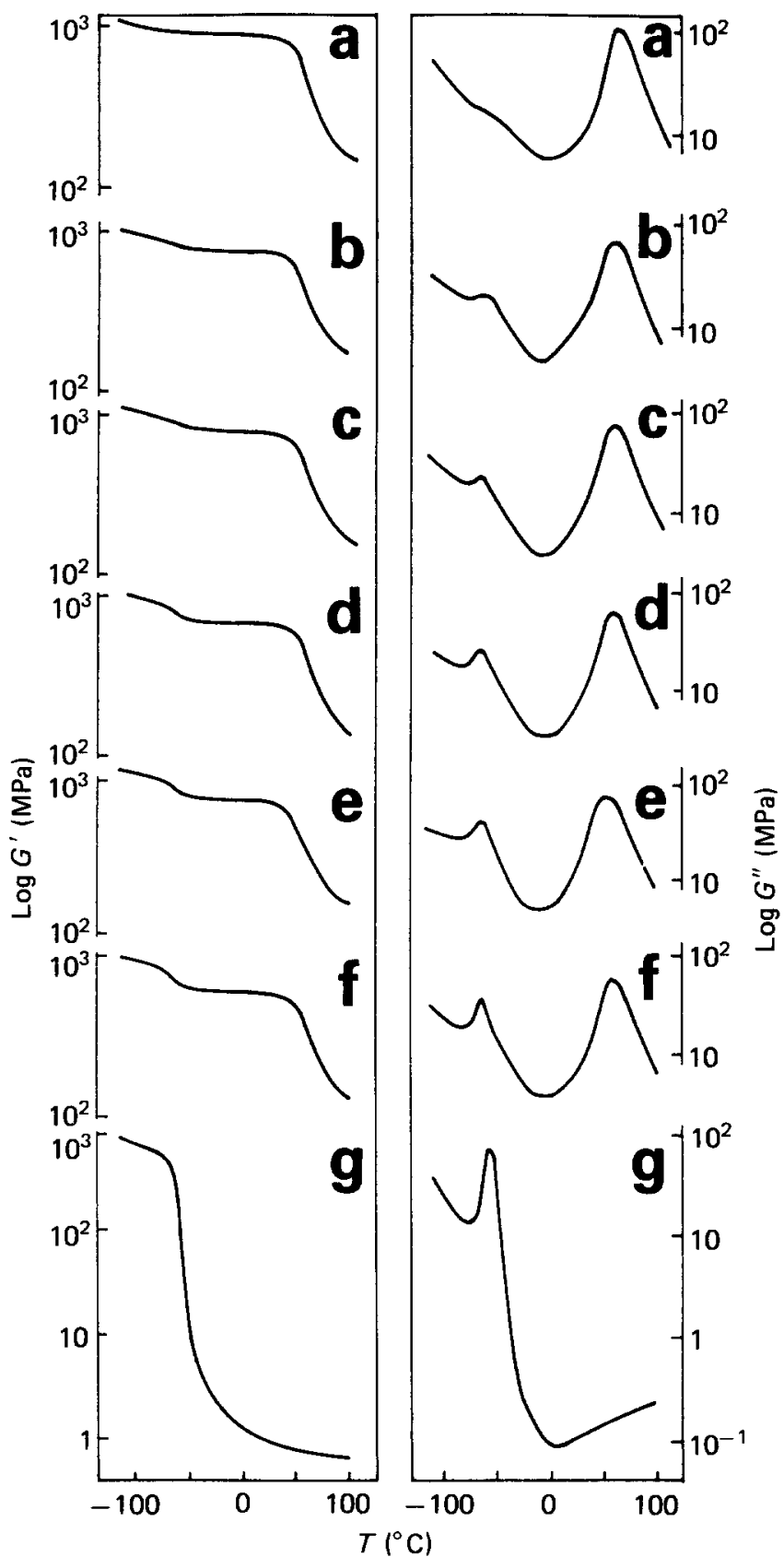

Figure 3 Shear modulus $G^{\prime}$ and loss modulus $G^{\prime \prime}$ as a function of temperature. (a) Nylon-6; (b) blend A2; (c) A3; (d) A4; (e) A5; (f) A6; (g) EPDM modified with $0.4 \mathrm{wt} \%$ maleic anhydride 


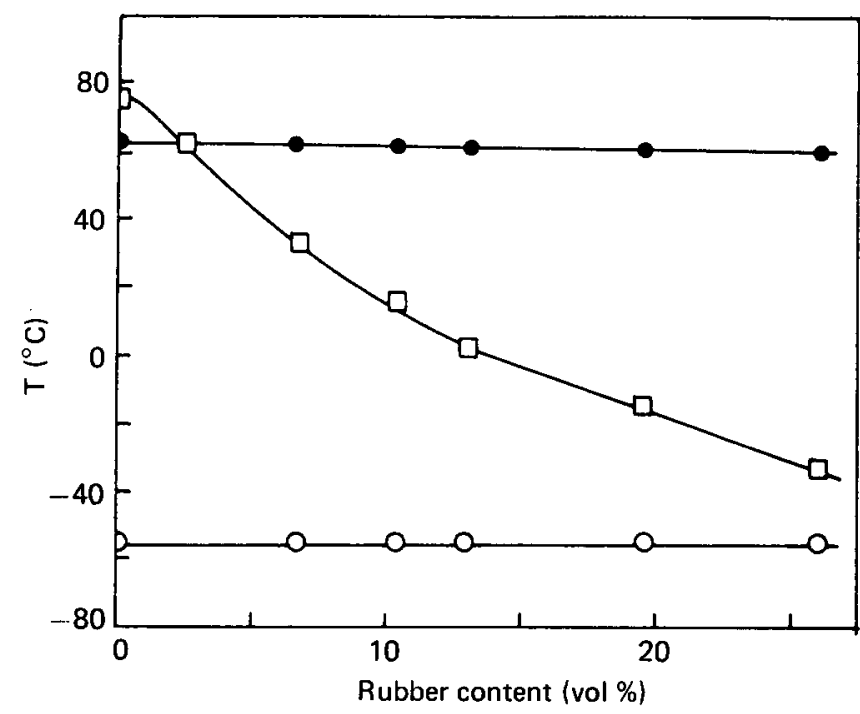

Figure 4 Brittle-tough temperature ( $\square$ ), $T_{\mathrm{g}}$ of the nylon matrix (O) and $T_{\mathrm{g}}$ of the dispersed rubber $(O)$ as functions of the rubber volume fraction for blends $A$

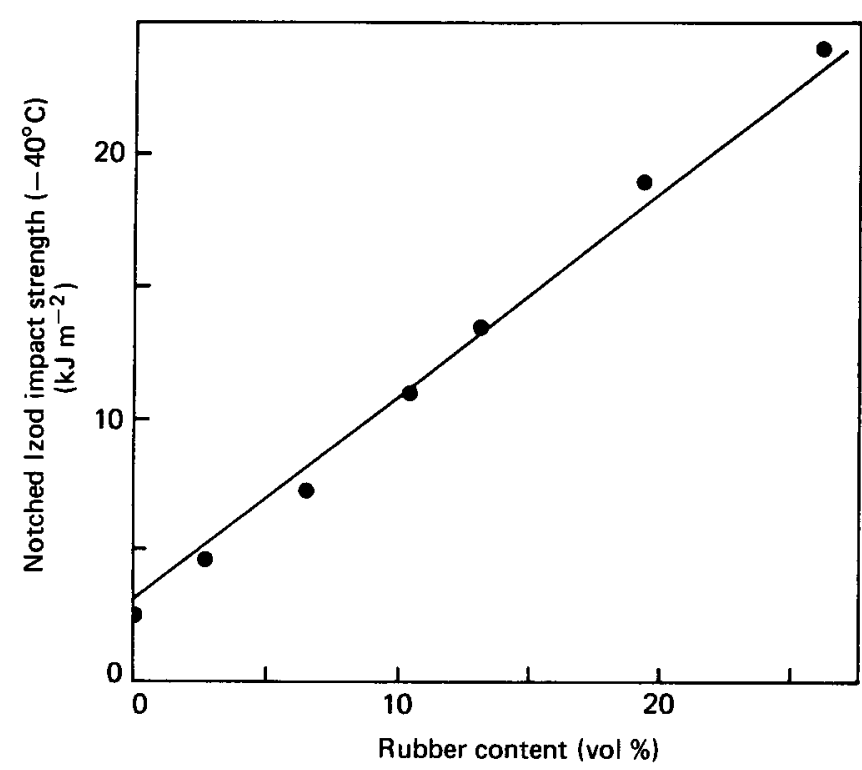

Figure 5 Notched Izod impact strength at $-40^{\circ} \mathrm{C}$ versus rubber volume fraction of blends $\mathrm{A}$. Particle size and interfacial adhesion are constant

shift with increasing rubber content, showing that the glass transition temperatures of the nylon matrix and of the dispersed rubber phase are not affected by the blending process, which means that there is complete phase separation.

In Figure 4 the BT-temperatures and $T_{\mathrm{g}}$ values of the $\mathrm{A}$ blends are given as a function of rubber volume fraction. The BT-temperature decreases strongly with rubber concentration and is independent of the $T_{\mathrm{g}}$ of nylon-6, which is constant. We have shown that, as a consequence, on wetting a nylon-rubber blend the $T_{\mathrm{g}}$ of the nylon is lowered, but the dispersion induced BT-temperature is not affected ${ }^{20}$. The dispersion-induced BT transition is clearly a second type of transition, independent of the $T_{\mathrm{g}}$ related brittle-tough transition.

It should be noted that the $T_{\mathrm{g}}$ values during the highspeed impact test are somewhat higher than the value obtained by the pendulum test, as can be described by the
Williams-Landel-Ferry equation for the rubber $^{21}$ and the Seitz and Balazs relation for the polyamide ${ }^{21}$. This will not essentially affect the conclusions.

In Figure 5, Izod impact strength at $-40^{\circ} \mathrm{C}$ of blends $\mathrm{A}$ is plotted against the volume fraction of rubber. The increase in impact energy when just $2 \mathrm{wt} \%$ rubber is added to nylon is already about a factor of 2 . A further remarkable gain in impact strength occurs when the rubber content increases. At this temperature all blends fracture in the brittle manner, even blend A.6, which has a notched impact strength of $23.9 \mathrm{~kJ} \mathrm{~m}^{-2}$.

Figure 2 shows also that the impact energies in the tough region seem to increase with increasing rubber content. Here it has to be noted that the tough fractures ( $>50 \mathrm{~kJ} \mathrm{~m}^{-2}$ ) could not be registered very accurately because many samples did not break completely. The reported impact energies are therefore minimum values.

In Figure 6 the notched impact strength versus temperature of series $B$ blends is given. The impact behaviour of these blends is rather poor since all BTtemperatures are $>45^{\circ} \mathrm{C}$. This could be the result of either the relatively large particle size or of a low interfacial adhesion, or the result of both.

\section{Variation of particle size}

In series $C$ blends, rubber properties and concentration as well as interfacial adhesion are constant and the effect of rubber particle size on the impact behaviour of the blends was studied. From Figure 7 it is clear that smaller particles shift the BT transition to lower temperatures. Also, the impact energy in the brittle region is higher when the average particle diameter is smaller.

Figure 8 plots the BT-temperatures of both C blends and blends $\mathrm{A} 6$ and $\mathrm{B} 6$, which have the same rubber content but a different concentration of maleic anhydride grafted on the rubber (and therefore probably a different interfacial adhesion to nylon-6), as a function of average particle diameter. The BT-temperatures of A6 and B6 are roughly in line with the BT-temperatures of the $\mathrm{C}$ blends, suggesting that interfacial adhesion, within the range studied, has no effect on the BT transition.

The modulus of EPDM starts to increase when the temperature approaches the $T_{\mathrm{g}}$ of the rubber and EPDM loses its rubber properties (Figure 3). A direct

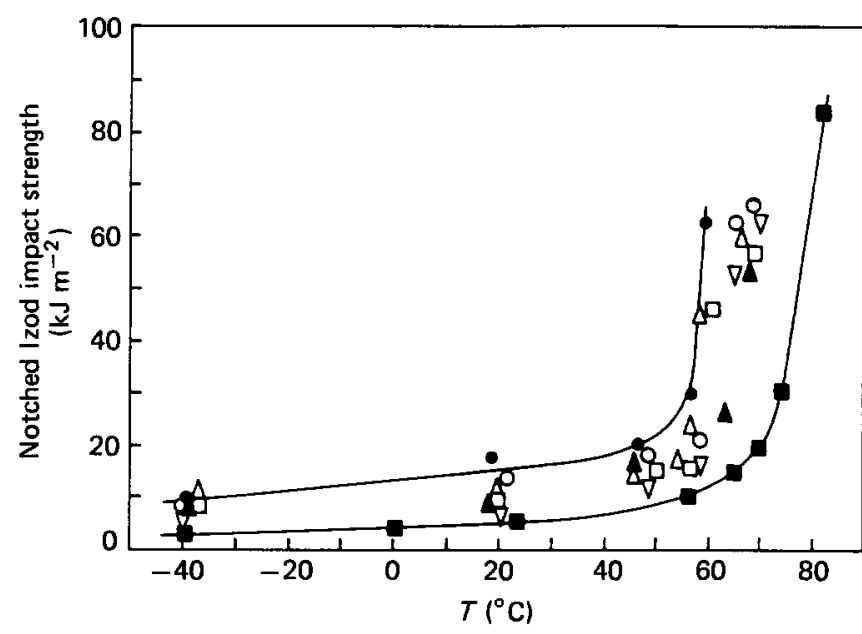

Figure 6 Notched Izod impact strength versus temperature of nylon-6 and blends B. $\square$, Nylon-6; $\nabla, \mathrm{B} 1 ; \Delta, \mathrm{B} 2 ; \square, \mathrm{B} 3 ; \mathrm{O}, \mathrm{B} 4 ; \triangle, \mathrm{B} 5 ; \mathrm{O}, \mathrm{B} 6$ 


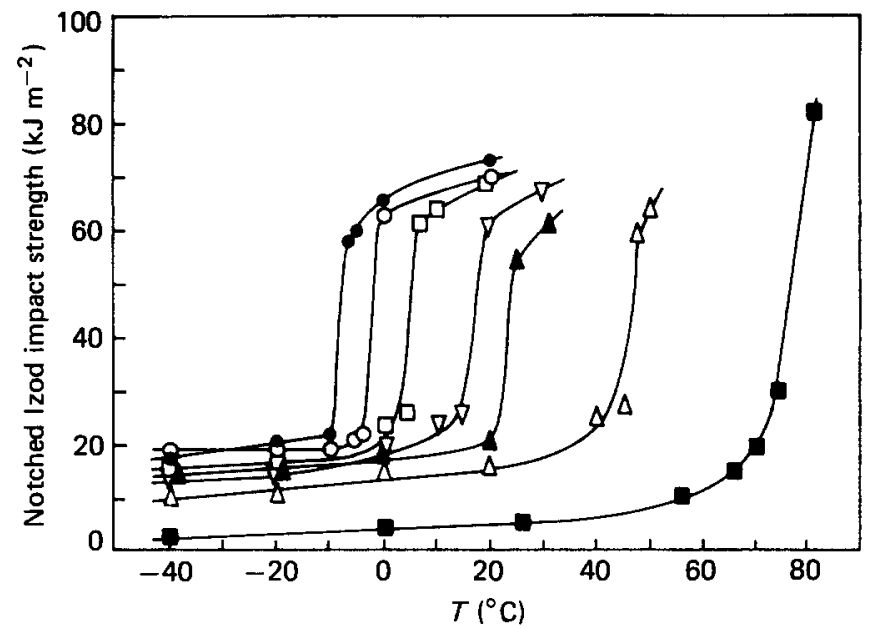

Figure 7 Notched Izod impact strength versus temperature of nylon-6 and blends $C$. Interfacial adhesion and rubber concentration $(26.1 \mathrm{vol} \%)$ are constant. Weight-average particle size $(\mu \mathrm{m}): \square$, nylon- $6 ; \triangle, 1.59$; $\triangle, 1.20 ; \nabla, 1.14 ; \square, 0.94 ; \bigcirc, 0.57 ; \bigcirc, 0.48$

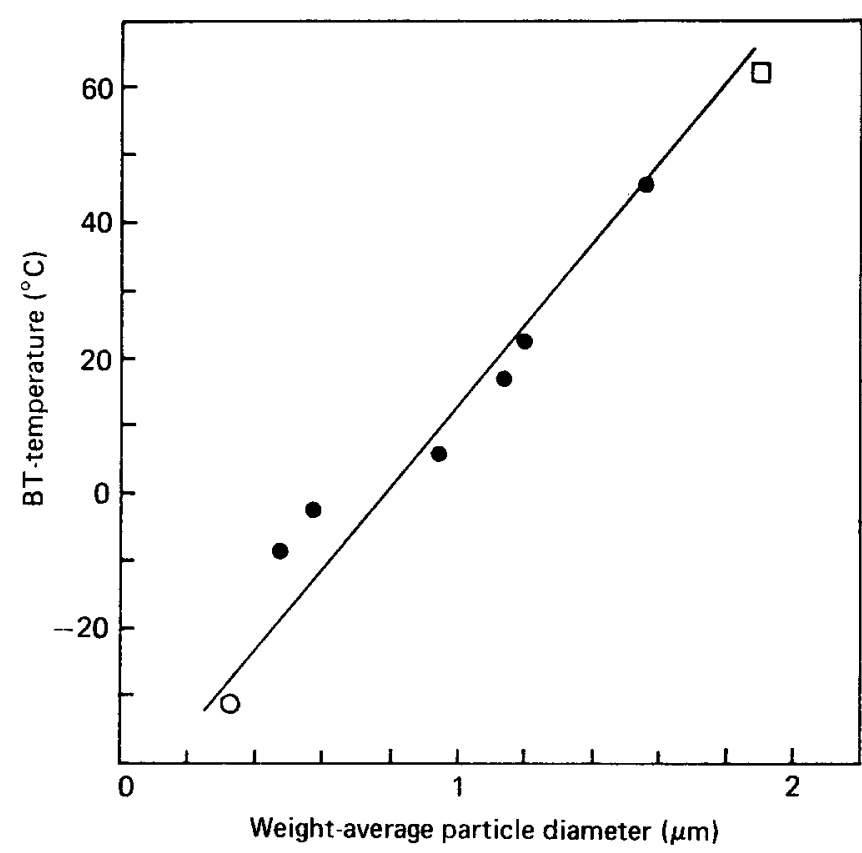

Figure 8 Brittle-tough temperature versus weight-average particle diameter. $O$, Blend $A 6 ; \square$, blend $B 6 ; 0$, blends $C$

consequence is that a lower limit in BT-temperature has to exist which will be higher than the $T_{\mathrm{g}}$ of the rubber. From Figure 4 it can be seen that blend A6 is near this limit and it is expected that neither a further increase in rubber content nor a further decrease in particle size will have a strong effect on the BT-temperature. An optimum particle size (which must be smaller than $0.3 \mu \mathrm{m}$ ) for this type of blend therefore cannot be found when using such high rubber concentrations.

\section{Yield stress and tensile modulus}

One of the functions of the rubber domains is to create stress concentrations, forcing the material to yield at lower applied stresses ${ }^{22,23}$.

In Figure 9 the normalized (dividing by yield stress of nylon-6) yield stresses of the A blends (excellent impact behaviour) and the $\mathrm{B}$ blends (poor impact behaviour) are plotted, measured with a tensile test with a drawing speed of $250 \% \mathrm{~min}^{-1}$. The yield stress of both series of blends decreases in the same way with increasing rubber content. The overall yield stress is apparently not affected by particle size or interfacial adhesion, while particle size was shown to have a strong effect on the BT transition. Rubber modification of polymers indeed induces a lowering of the overall yield stress. This reduction is not, however, the only criterion for toughening of nylons, since both tough and brittle blends show the same drop in yield stress with increasing rubber content.

The decrease of the yield stress does not follow the Ishai and Cohen equation $\sigma_{\mathrm{y}}=\sigma_{\mathrm{y}_{0}}\left(1-1.21 \phi^{2 / 3}\right)$ which is based on the effective area model described by Bucknall ${ }^{23}$.

Figure 10 demonstrates that the rubber in blends $\mathrm{A}$ and $B$ is well dispersed without inclusions since the Young's modulus decreases linearly with rubber content. The modulus also seems to be independent of particle size and interfacial adhesion.

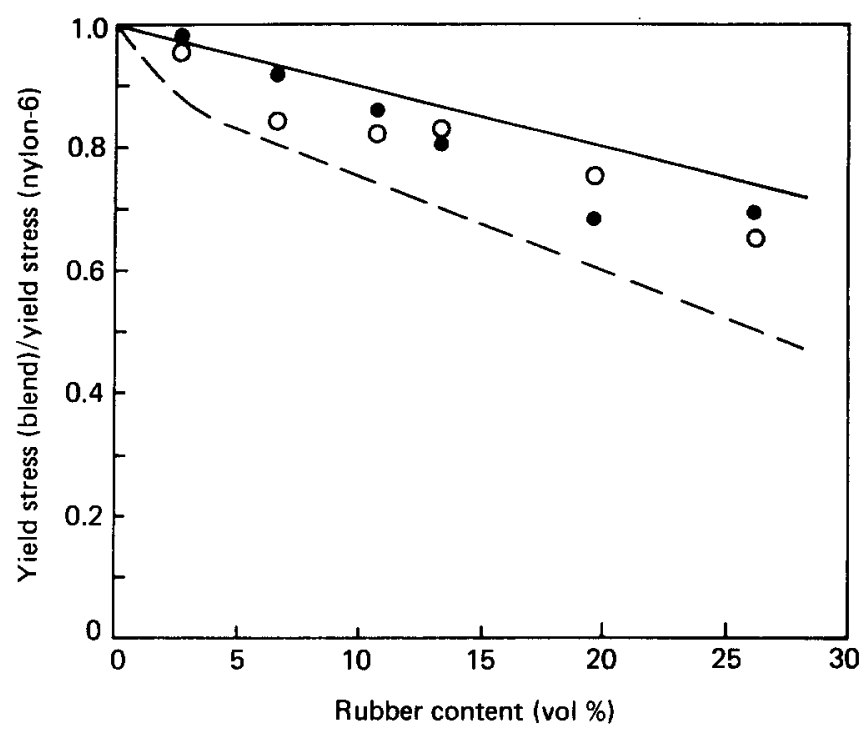

Figure 9 Normalized yield stress versus rubber volume fraction. Blends $\mathrm{A} ; \mathrm{O}$, blends $\mathrm{B}$

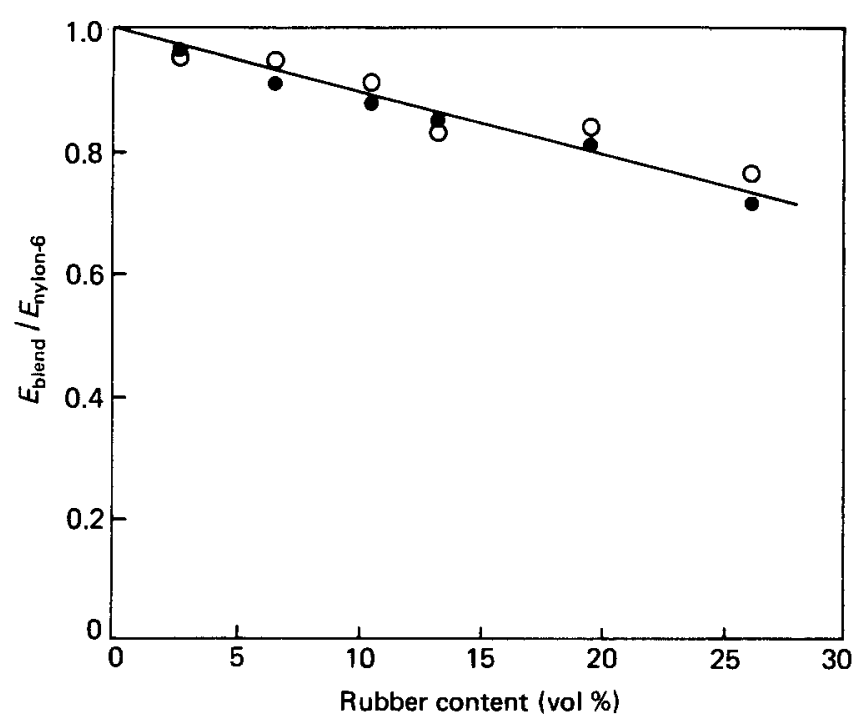

Figure 10 Normalized Young's modulus versus rubber volume fraction. $\mathrm{O}$, Blends $\mathrm{A} ; \mathrm{O}$, blends B 

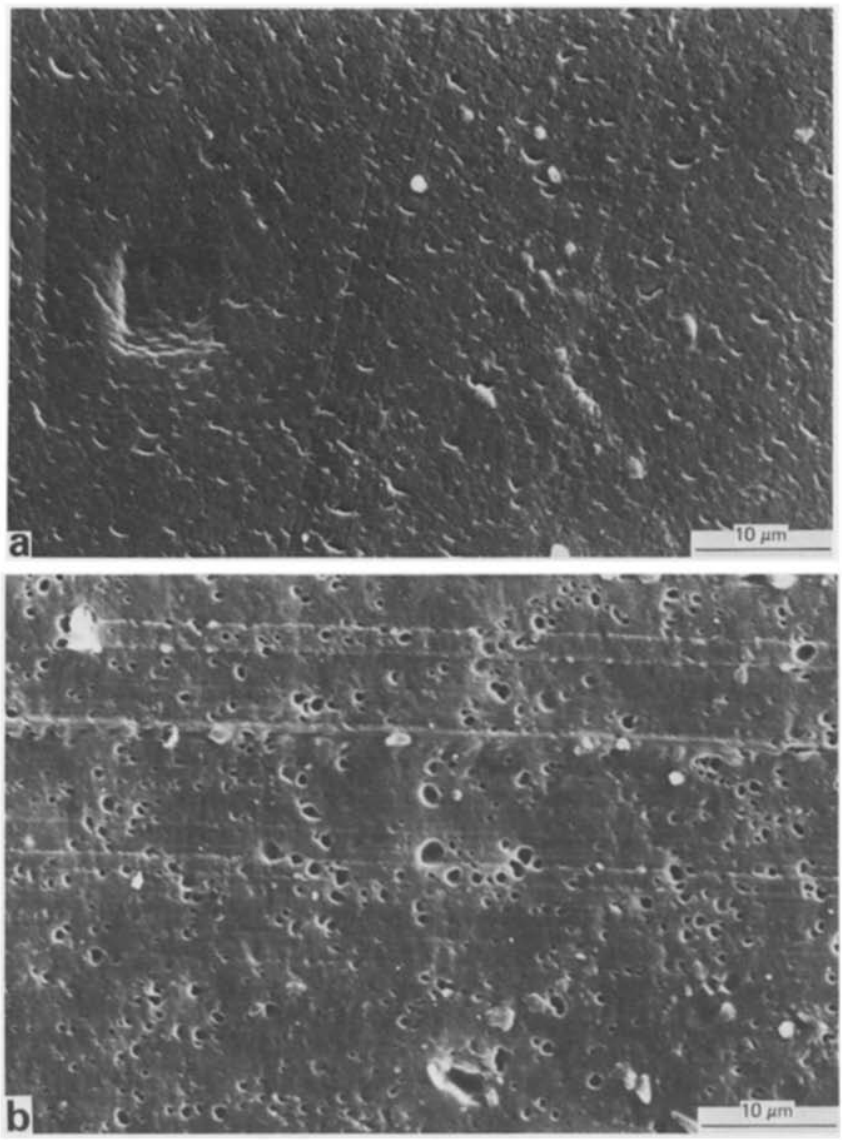

Figure 11 Scanning electron photomicrographs of blend A4. (a) Undeformed, (b) after a tensile test $\left(250 \% \mathrm{~min}^{-1}\right)$, showing magnification of the stress-whitened zone

\section{Deformation mode}

We used scanning electron microscopy (s.e.m.) to study the stress-whitened zone, which is the deformation and energy absorption zone, of a nylon-6/EPDM blend after a tensile test and then compared it with an undeformed sample (Figure 11). No crazing was observed in the deformed sample, only holes referring to voiding in or around the rubber particles. Ramsteiner ${ }^{24}$ also did not observe crazing during plastic deformation of nylonrubber blends, but only shear yielding in the matrix and voiding inside the rubber particles.

Impact fracture surfaces were studied by either etching the rubber with boiling xylene or staining the rubber with $\mathrm{OsO}_{4}$. Typical brittle surfaces have a stress-whitened zone immediately behind the notch, followed by a crack path where practically no matrix yielding took place. Figure 12 shows that rubber particles are in a brittle fracture path since etching of the rubber with boiling xylene leaves hemispherical holes in the surface. After staining the rubber with $\mathrm{OsO}_{4}$, rubber particles can indeed be seen in and on the surface.

Figure 13 shows the deformed surface after a tough fracture. Voids can be observed with diameters a few times greater than the rubber particle diameter. No difference is seen between the original fracture surface and the fracture surface etched with boiling xylene. In contrast to the results of $\mathrm{Wu}^{5}$, we were able to observe rubber particles on a tough fracture surface when they were stained with $\mathrm{OsO}_{4}$. Delamination of the EPDM particles from the matrix seems to be the cause of voiding during the plastic deformation process.

\section{Model study of the brittle-tough transition}

$\mathrm{Wu}^{7}$ introduced the critical interparticle distance $I D_{\mathrm{c}}$ as the only determining material parameter for the BT transition.

$$
I D_{c}=\mathrm{d}_{c}^{*}\left[(\pi / 6 \phi)^{1 / 3}-1\right]
$$

where $d_{\mathrm{c}}=$ critical particle size and $\phi=$ rubber volume content.

From Figures 2,6 and 7 it is clear that $I D_{c}$ for Izod tests must be temperature dependent and an $I D_{\mathrm{c}}$-temperature diagram can be constructed.

In Figure 14 the $I D_{\mathrm{c}}$ values calculated for the three types of blend are compared. The values show roughly the
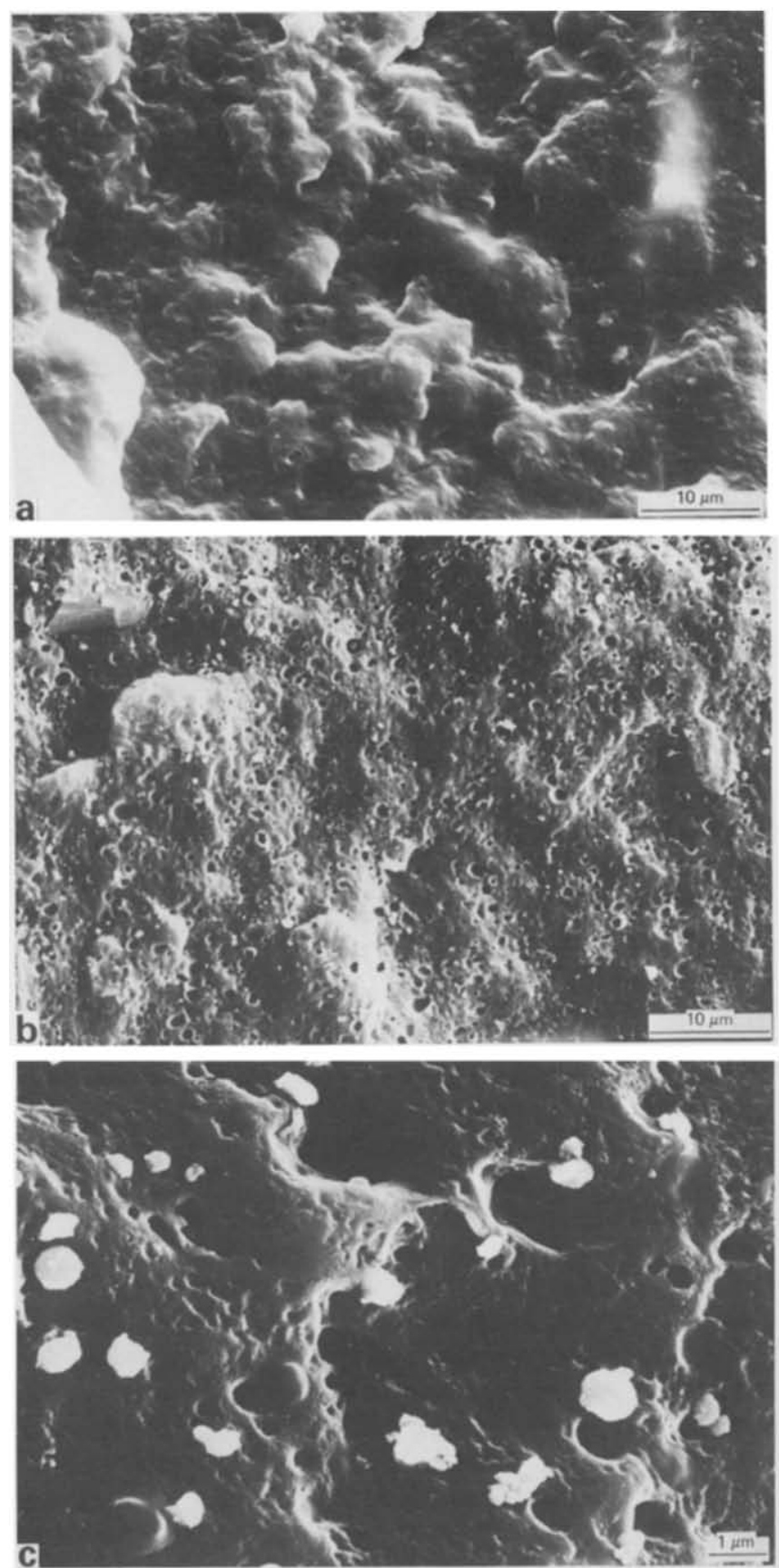

Figure 12 Brittle fracture surfaces of blend A4, studied by s.e.m. The notched impact test was at $-40^{\circ} \mathrm{C}$. (a) Original fracture surface; (b) fracture surface etched with boiling xylene; (c) fracture surface stained with $\mathrm{OsO}_{4}$ 

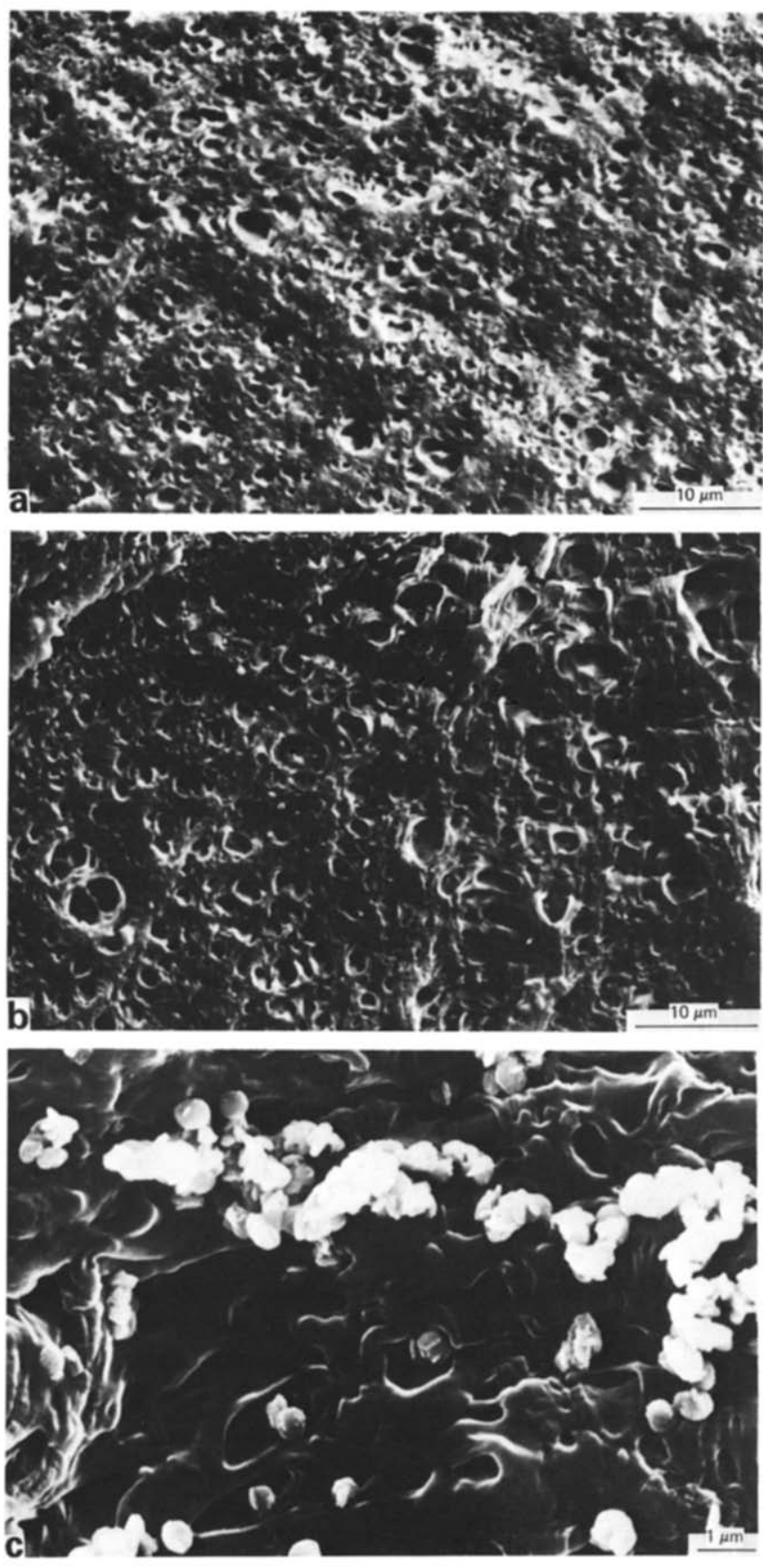

Figure 13 Tough fracture surfaces of blend A4, studied by s.e.m. The notched impact test was at $23^{\circ} \mathrm{C}$. (a) Original fracture surface; (b) fracture surface etched with boiling xylene; (c) fracture surface stained with $\mathrm{OsO}_{4}$

same temperature dependence. When the temperature approaches the $T_{\mathrm{g}}$ of nylon-6 the BT transition is no longer induced by rubber particles and a critical interparticle distance has no meaning.

Since different levels of interfacial adhesion can be expected in the three types of blend, Figure 14 also indicates that interfacial adhesion has, within the range studied, no influence on the BT transition. This result could give some support to Wu's conclusion that a van der Waals adhesion between the two phases is enough for the toughening effect.

A more extensive study on the importance of interfacial adhesion for impact behaviour of nylon-rubber blends is being executed ${ }^{25}$. Our results seem to support Wu's interparticle distance model. According to $\mathrm{Wu}$, the

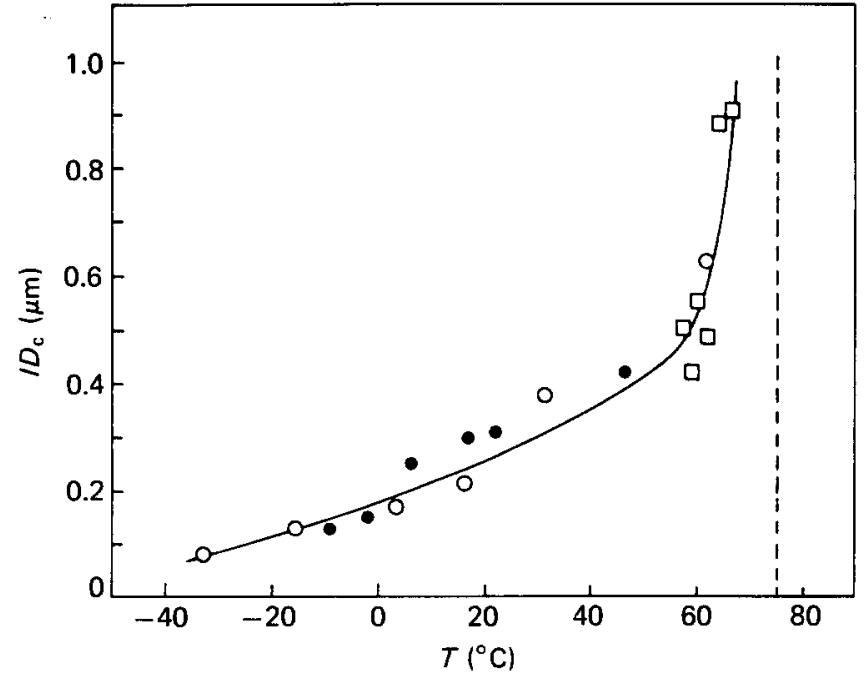

Figure 14 Interparticle distance versus the brittle-tough temperature. $O$, Blends $A ; \square$, blends B; $O$, blends $C$

interparticle distance model finds its origin in the possibility of an overlap of stress fields around neighbouring particles. However, blends with a low rubber concentration and small particle size (and BTtemperatures above room temperature) have $I D_{\mathrm{c}}$ values which are so great compared to the particle diameter that the effect of stress field overlap is not relevant.

It is known that the stress concentration factor drops to nearly 1 when the distance from a sphere with diameter $d$ is $1 / 2 * d$ (refs. 26 and 27). For example, blend A2 $\left(\phi=0.06 ; d_{\mathrm{w}}=0.38 \mu \mathrm{m}\right)$ has an interparticle distance of $0.40 \mu \mathrm{m}$ and therefore a BT-temperature of $31^{\circ} \mathrm{C}$. From a mechanical point of view, stress field interaction never can take place with this blend morphology. Although our results fit in the interparticle distance model, the physical explanation in terms of stress field overlap must be questioned. For the same reason, the validity of the interaction model of $\mathrm{Hobbs}^{6}$ is disputable.

\section{CONCLUSION}

Introducing rubber particles into nylon- 6 creates a sharp brittle-tough transition which is independent of the glass transition temperature of the nylon matrix. The brittletough transition temperature for notched Izod impact tests shifts to a lower value, while brittle and tough impact energies are greater when rubber content is increased and particle size is decreased. With 26.1 volume $\%$ rubber, the lowest limit for the BT-temperature was almost reached: $-32^{\circ} \mathrm{C}$. The optimum particle size must be smaller than $0.32 \mu \mathrm{m}$.

Interfacial adhesion and particle size do not affect yield stress and Young's modulus of nylon-rubber blends. Lowering of the yield stress is, therefore, not the only determining factor for toughening in nylons.

During plastic deformation voiding, by delamination of the EPDM-rubber particles from the matrix, and matrix yielding takes place. No crazing was observed as a part of the plastic deformation process. The interparticle distance model of $\mathrm{Wu}$ is shown to be applicable, although its physical explanation is questioned. 


\section{ACKNOWLEDGEMENTS}

This work is part of the research programme of the University of Twente and was financially supported by the Netherlands Technology Foundation (STW) and by the DSM Research Company.

\section{REFERENCES}

Flexman, E. A. Polym. Eng. Sci. 1979, 19, 564

Flexman, E. A. Kunststoffe 1979, 3, 172

Fahnler, F. and Merten, J. Kunststoffe 1985, 3, 157

Jang, B. Z., Uhlmann, D. R. and Vander Sande, J. B. J. Appl. Polym. Sci. 1984, 29, 3409

5 Wu, S. J. Polym. Sci., Polym. Phys. Edn. 1983, 21, 699

6 Hobbs, S. Y., Bopp, R. C. and Watkins, V. H. Polym. Eng. Sci. $1983,23,1983$

7 Wu, S. Polymer 1985, 26, 1855

8 Merle, G., Yong-Sok, O., Pillot, C. and Sautereau, H. Polym. Testing 1985, 5, 37

9 Adams, G. C. and Wu, T. K., 'Fáilure of Plastics', Hanser Publishers, Munich, 1986, p. 144

10 Polato, F.J. Mater. Sci. 1985, 20, 1455

11 Rupprecht, L., 'Konstruieren mit thermoplastische kunststoffen', VDI Verlag, Dusseldorf, 1986, p. 19

12 Cimmino, S., D'Orazio, L., Greco, R., Maglio, G., Malinconico,
M., Mancarella, C., Martuscelli, E., Palumbo, R. and Ragosta, G. Polym. Eng. Sci. 1984, 24, 48

13 Gale, G. M. Plastics Rubber Process. Appl. 1982, 2, 347

14 Hooiveld, W., MSc Thesis, University of Technology Twente, 1985

15 Bach, G. 'Quantitative Methods in Morphology', Springer Verlag, Berlin, 1967, p. 23

16 Elmendorp, J. J., PhD Thesis, University of Technology, Delft, 1986

17 Wu, S. 'Proceedings Polyblends 1986', Montreal

18 Ogorkiewicz, R. M. 'Engineering Properties of Plastics', John Wiley and Sons, New York, 1970

19 Vieweg, R. and Muller, A. 'Kunststoffe Handbuch: Part VI. Polyamide', Carl Hanser Verlag, Munich, 1966

20 Gaymans, R. J., Borggreve, R. J. M. and Spoelstra, A. B. Polymer in press

21 Van Krevelen, D. W. and Hoftyzer, P. J., 'Properties of Polymers', Elsevier Scientific Publishing Company, Amsterdam, 1976

22 Kinloch, A. J. and Young, R. J. 'Fracture Behaviour of Polymers', Elsevier Applied Science Publishers, London, 2nd Edn., 1985

23 Bucknall, C. B. Adv. Polym. Sci. 1978, 27, 121

24 Ramsteiner, F. and Heckmann, W. Polymer 1985, 26 (Commun.), 199

25 Borggreve, R. J. M. and Gaymans, R. J., in preparation

26 Oxborough, R. J. and Bowden, P. B. Phil. Mag. 1974, 30, 171

27 Matsuo, M., Wang, T. T. and Kwei, T. K. J. Polym. Sci. (A-2) $1972,10,1085$ 